\title{
Giving Social Support at Work May Reduce Inflammation on Employees Themselves: A Participatory Workplace Intervention Study Among Japanese Hospital Nurses
}

\section{Tsukumi Tondokoro}

International University of Health and Welfare Faculty of Medicine Graduate School of Medicine: Kokusai Iryo Fukushi Daigaku Igakubu Daigakuin Igaku Kenkyuka Igaku Senko https://orcid.org/00000002-0888-6737

\section{Akinori Nakata ( $\square$ nakataa-tky@umin.ac.jp)}

International University of Health and Welfare https://orcid.org/0000-0003-3008-4615

\section{Yasumasa Otsuka}

University of Tsukuba: Tsukuba Daigaku

\section{Nobuyuki Yanagihara}

Kyushu Nutrition Welfare University: Kyushu Eiyo Fukushi Daigaku

\section{Ayumi Anan}

University of Occupational and Environmental Health School of Health Sciences: Sangyo Ika Daigaku

Sangyo Hoken Gakubu

\section{Hiromi Kodama}

University of Occupational and Environmental Health School of Health Sciences: Sangyo Ika Daigaku Sangyo Hoken Gakubu

\section{Noriaki Satoh}

University of Occupational and Environmental Health Japan: Sangyo lka Daigaku

\section{Research note}

Keywords: Giving social support, Inflammatory markers, Autonomic nervous activity, Job stress, Participatory workplace intervention, Hospital nurses

Posted Date: April 8th, 2021

DOl: https://doi.org/10.21203/rs.3.rs-384296/v1

License: (c) (i) This work is licensed under a Creative Commons Attribution 4.0 International License. Read Full License 


\section{Abstract}

\section{Objective}

Previously, we reported that the participatory workplace intervention was effective in reducing stressrelated inflammatory markers, i.e., interleukin-6, among 31 Japanese female nurses. During the analysis, we recognized that our intervention might have increased prosocial behaviors like giving social support to others in some participants. Based on this assumption, we ran a secondary analysis, which examined the effect of giving social support to others on inflammatory markers, autonomic nervous activity, and perceived job stress before and after a 5-month intervention. We divided participants into two groups; those who had increased scores on giving social support after the intervention (Group 1, $n=13$ ), and those who had decreased/unchanged in the scores (Group 2, $n=17$ ). Friedman test was used to examine the changes in outcome measures by the group.

Results

Group 1 showed significant decreases in interferon- $\gamma$, interleukin-6, and interleukin-12/23p40 immediately after the intervention, while interleukin-12/23p40 remained stably decreased three months later; Group 2 did not show changes in these markers. No significant changes were observed regarding autonomic nervous activity and perceived job stress. This study presented a significant insight that giving social support at work may provide health benefits towards employees themselves, via decreasing inflammation in the body.

\section{Introduction}

Organizational-level participatory workplace intervention, which aims to improve work environment and employees' health, is more likely to produce sustainable effects than interventions targeting an individual because employees take an active part in identifying problems and giving possible solutions by themselves [1, 2]. In our previous study, we reported that a 5-month lasting organizational-level participatory workplace intervention was effective in reducing stress-related inflammatory markers as represented by interferon (IFN)- $\gamma$, interleukin (IL)-6, and IL-12/23p40, and IL-15 among 31 Japanese female nurses [3]. During this course, we recognized that our intervention might have stimulated prosocial helping behaviors like giving social support to others in some participants. Based on this assumption, we decided to run a secondary analysis focusing on giving social support and physiological responses.

It is well documented that social support acts as a stress buffer, which contributes to improving mental and physical health [4-6]. With regard to physiological markers, a number of studies reported the existence of positive associations between receiving/perceived social support and inflammatory markers and autonomic nervous activities (ANA) [7-10]. These studies mainly focused on receiving social support at work rather than giving social support. There is a lack of evidence on the effects of giving social support on physiological outcomes. Although limited studies on giving social support, two intervention studies examined the effects of giving social support on physiological responses (inflammatory markers, 
heart rate, blood pressure, salivary alpha-amylase, and salivary cortisol) among healthy individuals [11, 12]. These studies revealed that giving social support contributed to decreasing inflammatory markers, systolic blood pressure, and salivary alpha-amylase [11, 12]. However, the study settings were experimental, i.e., the intervention was to imagine someone whom participants wanted to support and write a supporting letter to him/her, etc. To the best of our knowledge, no organizational-level studies to date have examined giving social support and physiological outcomes in a work setting.

Therefore, the present study aimed to explore how changes in giving social support to others at work affect physiological responses among Japanese female nurses. We hypothesized that those who increased giving social support by the intervention would have a positive effect on inflammation and ANA; those who had increased scores on giving social support after the intervention exerted decreased inflammatory makers and ANA to healthier status compared to those who had no change or decreased scores.

\section{Methods}

\section{Participants and procedure}

We carried out a participatory workplace improvement intervention [13-22] from August 2017 to February 2018. Briefly, the participatory workplace improvement intervention is that employees at the workplace actively take part in identifying workplace problems, find feasible actions/solutions, and work towards improvement. We recruited nurses $(n=144)$ working at a hospital with 150 beds in the southern part of Japan. A total of 36 nurses agreed to participate in this study. We conducted evaluations before the intervention for baseline ( $\mathrm{T} 1$ ), within a week after the end of the intervention to assess immediate effects (T2), and 3 months after the end of the intervention to assess prolonged and lasting effects (T3). We excluded participants who became pregnant during the study period $(n=1)$, missed evaluations $(n=3)$ and had incomplete responses in giving social support scores $(n=1)$. A male participant $(n=1)$ was also excluded because of possible sex differences in outcome measures. Therefore, a total of 30 female nurses were submitted to the final analysis.

\section{Measures}

\section{Sociodemographic, lifestyle, health, and occupational conditions}

We used the self-administered questionnaire to assess participants' sociodemographic and job-related characteristics including social support at work and perceived psychosocial job stress.

\section{Giving social support}

In the questionnaire, we included questions of 'giving' social support to others at work, which we modified from 'receiving' social support in the Brief Job Stress Questionnaire [23]; "How much help do you provide 
to the following people?", "How much are you relied on by the following people?", "How well do you listen to the following people when they ask for advice on personal matters?". Participants answered each question by "superiors", "co-workers", and "subordinates" with a four-point scale ( $1=$ extremely to $4=$ not at all). Cronbach's alphas for these items were $>0.630$ at all-time points.

\section{Inflammatory markers}

We used serum interferon (IFN)-y, interleukin (IL)-6, tumor necrosis factor (TNF)-a, IL-12/23p40, IL-15, IL27, and high-sensitivity C-reactive protein (hs-CRP) as inflammatory markers. On the evaluation days, participants (nurses) brought their blood samples which collected between $2 \mathrm{pm}$ and $5 \mathrm{pm}$ in gammarays sterilized polyethylene-terephthalate tubes containing serum separating gel and coagulation accelerant (silica particles). We stored the samples in a cooler box $\left(0-5^{\circ} \mathrm{C}\right)$ and transported them to our laboratory twice a day by $4: 30 \mathrm{pm}$ and $7: 30 \mathrm{pm}$. In the laboratory, we centrifugalized them with 2,400 rpm for 10 minutes to extract $500 \mu \mathrm{L}$ of the serum and deep-freezed $\left(-20^{\circ} \mathrm{C}\right)$ until the analysis. The level of inflammatory markers was assessed with the Enzyme Immunoassay or Chemiluminescent Enzyme Immunoassay with MESOTM QuickPlex SQ 120 (Meso Scale Diagnostic, LCC, Rockville, USA) by the analyzing company, Life Science Institute Medience Corporation, Japan. The minimum detectable level for IFN-y, IL-6, TNF-a, IL-12/23p40, IL-15, IL-27, and hs-CRP was $0.2 \mathrm{pg} / \mathrm{ml}, 0.06 \mathrm{pg} / \mathrm{ml}, 0.04 \mathrm{pg} / \mathrm{ml}, 15.0$ $\mathrm{pg} / \mathrm{ml}, 2.0 \mathrm{pg} / \mathrm{ml}, 8 \mathrm{pg} / \mathrm{ml}$, and $0.004 \mathrm{mg} / \mathrm{dl}$, respectively. We calculated the values lower than them into the minimum detectable level $/ \sqrt{ } 2$, as it was described elsewhere [24].

\section{Autonomic nervous activity (ANA)- Heart rate variability (HRV)}

We utilized an electrocardiograph device, Silmee Bar Type Lite (Silmee; Tokyo Denki Kagaku, Tokyo, Japan) to measure heart rate variability (HRV). Silmee automatically calculates HRV by the power spectral analysis and measures 3 sympathetic nervous activity (SNA); low-frequency HRV/total frequency HRV (standing position), mean R-R interval/R-R interval per minute (standing position), and mean R-R interval (supine-stand position), and 3 parasympathetic nervous activity (PNA) parameters; mean R-R interval (supine position), high-frequency HRV/total frequency HRV (supine position), and the standard deviation of R-R intervals (SDRR) (supine position). Silmee also calculates SNA/PNA. We measured participants' autonomic nervous activities in two rooms at the hospital between $2 \mathrm{pm}$ and $5 \mathrm{pm}$ to adjust in-day fluctuation.

\section{Statistical Analyses}

Based on the total giving social support score at each time-point, we divided participants into two groups; those who had increased scores on giving social support to others after the intervention (Group 1, $n=13$ ), and those who had decreased/unchanged in the scores (Group 2, n =17). After the confirmation of nonGaussian distribution with the Shapiro-Wilk test, we applied the Friedman test to examine changes in inflammatory markers, autonomic nervous activity, and perceived job stress by the group. We analyzed data using IBM SPSS Statistics for Windows, version 25.0 (IBM Corp., Chicago, IL, USA), and the level of significance was set at $p<0.05$. 


\section{Results}

Table 1 shows the baseline characteristics of female nurse participants. The median age of participants was 37.0 years old for Group 1 and 38.0 years old for Group 2. More than $60 \%$ of participants were not married in both groups. Except for department $\mathrm{G}$ (nursing department), 4 to 6 nurses in each unit participated. More than half of the participants worked for the day shift in both groups. Over $60 \%$ of participants had 6 or more hours of sleep on workdays in both groups. Most of them in both groups were under regular menstrual cycle (> 76.5\%).

Table 2 presents the changes of physiological markers overtime in Group 1. IFN- $\gamma(p=0.005)$, IL-6 ( $p=$ 0.018), and IL-12/23p40 ( $p=0.018)$ were decreased at T2 compared to T1. IL-12/23p40 was decreased at T3 compared to T1 $(p=0.013)$. The overall changes of TNF-a were also significant $(p=0.021)$, but it was insignificant with Bonferroni-adjusted pairwise tests. No significant decreases were found in ANA.

Table 3 shows the changes of physiological markers in Group 2 over time. Neither blood inflammatory markers nor ANA showed insignificant decreases.

Tables S1 and S2 (Additional files 1 and 2) present the changes of perceived job stress over time by the groups. There were no significant decreases with Bonferroni-adjusted pairwise tests in both groups.

\section{Discussion}

This study examined the effect of giving social support to others on inflammatory markers, autonomic nervous activity, and perceived job stress before and after the participatory workplace intervention among Japanese female nurses. As we hypothesized, the group with increased levels of giving social support (Group 1) showed significant post-intervention decreases in inflammatory markers (IFN- $\gamma$, IL-6, and IL12/23p40), while another group with decreased/unchanged levels of giving social support (Group 2) did not show such changes in these markers. ANA and perceived job stress did not show significant changes in both groups. This is one of the first studies to examine changes in giving social support to others at work after an organizational-level intervention using multiple physiological markers.

We observed decreases in inflammatory markers only in Group 1. Our finding is comparable with a past study regarding giving social support and inflammatory markers. A study by Moieni et al. reported that increases in giving social support levels are related to decreases in inflammatory markers [12]; a 6-week gratitude intervention resulted in decreases in the percentage of monocytes producing IL-6, TNF-a, and coproducing IL-6 and TNF-a via increases in support-giving among healthy middle-aged women. On the other hand, the control group did not lead to such changes. Our results also imply that giving social support to others at work may contribute to improving health by decreasing inflammatory markers on employees themselves.

Although several inflammatory markers had decreased in Group 1 after the intervention, another physiological measure (ANA) remained unchanged in the same group. The plausible explanation is that 
positive outcomes may emerge at different timing in each measure [3]. Past participatory workplace intervention studies did not also obtain positive effects simultaneously in all stress-related measures they used in the intervention group [20-22, 25], despite longer intervention period compared to our study. The various types of measures and timing of evaluation may have led to disaggregated results on inflammatory markers and ANA in Group 1.

In conclusion, this study presented a significant insight that increases in giving social support to others at work may have positive health effects on employees themselves via reducing inflammation in ones' body. Further studies with a better study design, i.e., randomized control design, are required to confirm the effects. Moreover, work remains to investigate the amount and timing of giving social support at work, and towards who (supervisor, family, etc.) provides social support is important in exerting beneficial effects on employees' health.

\section{Limitations}

- Small sample size

- No control group

- Other confounding factors might be influenced the results

- Data analysed by two separate groups due to the non-Gaussian distribution of the data.

\section{Abbreviations}

Group 1: the group of nurses with increased scores in giving support after the intervention; Group 2: the group of nurses with decreased/unchanged scores in giving support after the intervention; ANA:

Autonomic nervous activity; HRV: Heart rate variability; IFN: Interferon; IL: Interleukin; TNF: Tumor necrosis factor; hs-CRP: High sensitivity C-reactive protein; T1: baseline evaluation; T2: within a week after the intervention; T3: three months after the end of the intervention.

\section{Declarations}

\section{Acknowledgments}

We thank nurses who participated in this study and nurse representatives for help. We are also grateful to anyone who helped us conduct this study at this stage.

\section{Authors' contributions}

AN designed the study and TT, AN, and AA contributed coordination. TT, AN, YO, and AA facilitated recruitment, and TT, AN, and YO facilitated the intervention. TT, NY, HK, and NS contributed data collection and TT performed serum extraction. AN supervised TT on the data analysis and paper conduct. All authors read and approved the final manuscript. 


\section{Funding}

This study was supported by KAKENHI Grant Number 26671048 and $19 \mathrm{H} 01763$, and the Work-related Diseases Clinical Research Grant (160701-01, 170701-01, 180701-01) from the Ministry of Health, Labour and Welfare, Japan.

\section{Availability of data and materials}

The datasets used and/or analyzed during the current study are available from the corresponding author on reasonable request.

\section{Declarations}

\section{Ethics approval and consent to participate}

The study was reviewed and approved by the ethical committee of the International University of Health and Welfare (18-ml-002). This study was registered on the University Hospital Medical Information Network Clinical Trials Registry (UMIN000039836). We informed potential participants about the study aim, procedure, and confidentiality policy for individual information. Written informed consent was obtained from those who agreed to participate. At each time of the evaluation, they received a 1,000-yen gift card as a reward.

\section{Consent for publication}

Not applicable.

\section{Competing interests}

The authors declare that they have no competing interests.

\section{References}

1. Montano D, Hoven H, Siegrist J. Effects of organisational-level interventions at work on employees' health: a systematic review. BMC Public Health. 2014;14(1):135.

2. Lamontagne AD, Keegel T, Louie AM, Ostry A, Landsbergis PA. A systematic review of the job-stress intervention evaluation literature, 1990-2005. Int J Occup Environ Health. 2007;13(3):268-80.

3. Tondokoro T, Nakata A, Otsuka Y, Yanagihara N, Anan A, Kodama H, et al. Effects of participatory workplace improvement program on stress-related biomarkers and self-reported stress among university hospital nurses: a preliminary study. Ind Health. 2021. https://doi.org/10.2486/indhealth.2020-0176

4. Cohen S, Wills TA. Stress, social support, and the buffering hypothesis. Psychol Bull. 1985;98(2):31057.

5. Kawachi I, Berkman LF. Social ties and mental health. J Urban Health. 2001;78(3):458-67. 
6. Qiao S, Li X, Zhou Y, Shen Z, Stanton B. Attitudes toward evidence-based practices, occupational stress and work-related social support among health care providers in China: A SEM analysis. PLoS One. 2018;13(8):e0202166.

7. Nakata A. Psychosocial job stress and immunity: a systematic review. Methods Mol Biol. 2012;934:39-75.

8. Nakata A, Irie M, Takahashi M. Source-specific social support and circulating inflammatory markers among white-collar employees. Ann Behav Med. 2014;47(3):335-46.

9. Eguchi H, Watanabe K, Kawakami N, Ando E, Arima H, Asai Y, et al. Psychosocial factors at work and inflammatory markers: protocol for a systematic review and meta-analysis. BMJ Open. 2018;8(8):e022612.

10. de Looff PC, Cornet LJM, Embregts PJCM, Nijman HLI, Didden HCM. Associations of sympathetic and parasympathetic activity in job stress and burnout: A systematic review. PLoS One. 2018;13(10):e0205741.

11. Inagaki TK, Eisenberger NI. Giving support to others reduces sympathetic nervous system-related responses to stress. Psychophysiology. 2016;53(4):427-35.

12. Moieni M, Irwin MR, Haltom KEB, Jevtic I, Meyer ML, Breen EC, et al. Exploring the role of gratitude and support-giving on inflammatory outcomes. Emotion. 2019;19(6):939-49.

13. Shimomitsu T, Kobayashi A, Nakahara T, Iwata N, Kawakami N, Tsutsumi A, et al. Studies regarding improving mental health by workplace improvement (in Japanese). Japan: Occupational safey and health research group; 2005.

14. Japan National Personnel Authority. Procedure for workplace improvement (in Japanese). Japan: Japan National Personnel Authority; 2016.

15. Yoshikawa T, Yoshikawa E, Tsuchiya M, Kobayashi Y, Shimazu A, Tsutsumi A, et al. Development of evidenced-based medicine guidelines for improving the workplace environment by means of primary job stress prevention. Job Stress Res. 2013;20(2):135-45.

16. Magnavita N. Medical surveillance, continuous health promotion and a participatory intervention in a small company. Int J Environ Res Public Health. 2018;15(4):662.

17. Lavoie-Tremblay M, Bourbonnais R, Viens C, Vézina M, Durand PJ, Rochette L. Improving the psychosocial work environment. J Adv Nurs. 2005;49(6):655-64.

18. Yoshikawa E. Concept analysis of a participatory approach to occupational safety and health (in Japanese). Sangyo Eiseigaku Zasshi. 2013;55(2):45-52.

19. Schneider A, Wehler M, Weigl M. Effects of work conditions on provider mental well-being and quality of care: a mixed-methods intervention study in the emergency department. BMC Emerg Med. 2019;19(1):1.

20. Kobayashi Y, Kaneyoshi A, Yokota A, Kawakami N. Effects of a worker participatory program for improving work environments on job stressors and mental health among workers: a controlled trial. J Occup Health. 2008;50(6):455-70. 
21. Tsutsumi A, Nagami M, Yoshikawa T, Kogi K, Kawakami N. Participatory intervention for workplace improvements on mental health and job performance among blue-collar workers: a cluster randomized controlled trial. J Occup Environ Med. 2009;51(5):554-63.

22. Romanowska J, Larsson G, Eriksson M, Wikström BM, Westerlund H, Theorell T. Health effects on leaders and co-workers of an art-based leadership development program. Psychother Psychosom. 2011;80(2):78-87.

23. Shimomitsu T, Haratani T. Ministry of labour Heisei 11th health labour sciences research report: Studies regarding improving mental health by workplace improvement; Reliability of the brief job stress questionnaire and the reference values (in Japanese). Japan: Stress Evalation Research group; 2000. p. 126-38.

24. Hornung RW, Reed LD. Estimation of Average Concentration in the Presence of Nondetectable Values. App Occup Environ Hyg. 1990;5(1):46-51.

25. Uchiyama A, Odagiri Y, Ohya Y, Takamiya T, Inoue S, Shimomitsu T. Effect on mental health of a participatory intervention to improve psychosocial work environment: a cluster randomized controlled trial among nurses. J Occup Health. 2013;55(3):173-83.

\section{Tables}

Due to technical limitations, table 1,2 and 3 is only available as a download in the Supplemental Files section.

\section{Supplementary Files}

This is a list of supplementary files associated with this preprint. Click to download.

- TableS1group1BMCPercievedStress.pdf

- TableS2Group2BMCPercievedStress.pdf

- Table1BMCDemographics2.pdf

- Tables2group1BMCTONDOKORO.pdf

- Tables3group2BMCTONDOKORO.pdf 